\title{
Investigating Strain as a Biomarker for Atrial Fibrosis Quantified by Patient Cine MRI Data
}

\author{
Ahmed Qureshi ${ }^{1}$, Aditi Roy ${ }^{1}$, Henry $_{\text {Chubb }}{ }^{1}$, Adelaide de Vecchi ${ }^{1}$, Oleg Aslanidi ${ }^{1}$ \\ ${ }^{1}$ School of Biomedical Engineering and Imaging Sciences, King's College London, United Kingdom
}

\begin{abstract}
Atrial fibrillation $(A F)$ is responsible for deterioration of left atrial (LA) mechanical function. Sinus rhythm (SR) can be restored by terminating $A F$ using catheter ablation (CA) therapy. CA often targets fibrotic tissue by creating scar tissue which is similar to fibrosis. We propose the use of myocardial strain to identify regions of fibrosis and understand its role in atrial mechanics.

Patient-specific LA models were reconstructed from Cine and Late Gadolinium Enhanced (LGE) MRI data for two groups of patients: $A F$ and SR pre-CA. $L G E$ intensities represented atrial fibrosis and feature-tracking was applied on the Cine images to produce a series of $3 D$ deforming LA meshes. The myocardial area strain (MAS) was calculated as a measure of regional contractile ability. 24 regions of clinical interest were assigned for inter-and intra-patient comparisons on the effects of $C A$ and fibrosis on LA mechanical function.

Correlation was found between low strain and dense fibrosis in the LA posterior wall for both patient groups $\left(r_{s}=-0.74\right) . M A S$ increased (8.9\%) after CA in the AF group but decreased (10\%) in the SR group.

This study suggests that myocardial strain can be used as a biomarker for atrial fibrosis and also identifies the detrimental effect of intentional CA-induced damage to the $L A$ on its mechanical function.
\end{abstract}

\section{Introduction}

Atrial fibrillation (AF) is the most prevalent sustained arrhythmia worldwide and it leads to uncoordinated electrical activations which diminish left atrial (LA) mechanical function [1]. Moreover, AF-induced structural remodelling damages both electrical conduction pathways and mechanical contractibility through the accumulation of rigid collagen in the form of atrial fibrosis.

Catheter ablation (CA) therapy uses radiofrequency heating of atrial tissue to isolate arrhythmogenic tissue and terminate AF. CA succeeds at the expense of creating scar tissue - another form of pathological fibrosis [2].

Late gadolinium enhanced (LGE) MRI has been established as a tool for myocardial fibrosis assessment. Increased uptake of gadolinium in fibrotic tissue can be used to measure AF progression and improve long-term prognoses [3]. However, this approach requires sophisticated image processing and can result in highly variable results between clinical centres.

Myocardial strain is an increasingly common clinical parameter used to quantify regional contractile ability and cardiac function; however, standard 2D ultrasound-based strain assessment techniques are not suitable for application to the 3D atria, prompting a search for innovative solutions using AF patient MRI data.

In this study, we propose a novel approach for calculating LA strain to evaluate altered mechanical function due to fibrosis induced by $\mathrm{AF}$ and $\mathrm{CA}$, which may help personalise the management of AF.

\section{Methods}

Patient-specific LA models with fibrosis maps were reconstructed from MRI and feature-tracking was applied for strain calculation. Assessment of the impact of fibrosis on LA strain was performed by unwrapping the 3D models to a standardised 2D disk representation.

\subsection{D Model Generation}

Patient-specific LA models were generated by manual segmentation of MRI data acquired by Chubb et al. [3, 4]. Imaging of AF patients due to undergo pulmonary vein (PV) isolation CA was performed on a 1.5T Philips Ingenia scanner pre- and 3 months post-ablation. Patient datasets $(\mathrm{N}=8)$ were selected for strain and fibrosis processing in two groups, $\mathrm{AF}(\mathrm{N}=3)$ and $\mathrm{SR}(\mathrm{N}=5)$. Patients in the AF group were imaged while in AF (pre$\mathrm{CA}$ ) and in SR (post-CA), whereas the SR group patients were in SR during both (pre- and post-CA) scans.

Temporally varying whole heart Cine MRI data was chosen for calculation of LA strain. The images had inplane axial resolution of $1.4 \times 1.4 \mathrm{~mm}^{2}$, slice thickness of $10 \mathrm{~mm}$ and temporal resolution of 50 time-steps per cycle. LGE images to reconstruct fibrosis were acquired using a 3D inversion-recovery spoiled gradient echo MRI sequence with image resolution of $1.3 \times 1.3 \times 4 \mathrm{~mm}^{3}$.

Manual segmentation of Cine and LGE data generated anatomical models of the LA endocardium at end systole. 


\subsection{Atrial Fibrosis Quantification}

Atrial fibrosis reconstruction was achieved by first mapping raw LGE image signal intensities to the 3D segmentations. A maximum intensity projection technique assigned the signal intensity from a point on the LGE image to its corresponding location on the endocardial surface model [4]. An example of the mapped LGE intensity distribution is shown in Figure 1A.

Quantification of LA fibrosis from raw LGE intensity distributions required an additional standardised solution to segment regions of fibrotic tissue [5]. While the model shown in Figure 1A provides a useful visualisation tool, this representation is not directly meaningful due to the variability of arbitrary unit value ranges of LGE intensity between patients and studies. The calculation of an image intensity ratio (IIR) for each voxel of the 3D LA provides a reliable representation of atrial fibrosis. The IIR is calculated by division of the voxel intensity by the mean blood pool intensity determined from the LGE image. Based on the IIR, each voxel was reassigned a value of 5 for dense fibrosis (IIR > 1.24), 0 for healthy tissue (IIR < $1.08)$ and between 1 and 4 for border zones $(1.08 \leq \mathrm{IIR} \leq$ 1.24). This labelling protocol, as shown in Figure 1B, enables improved visualisation and comparability of atrial fibrosis between pre- and post-CA images.
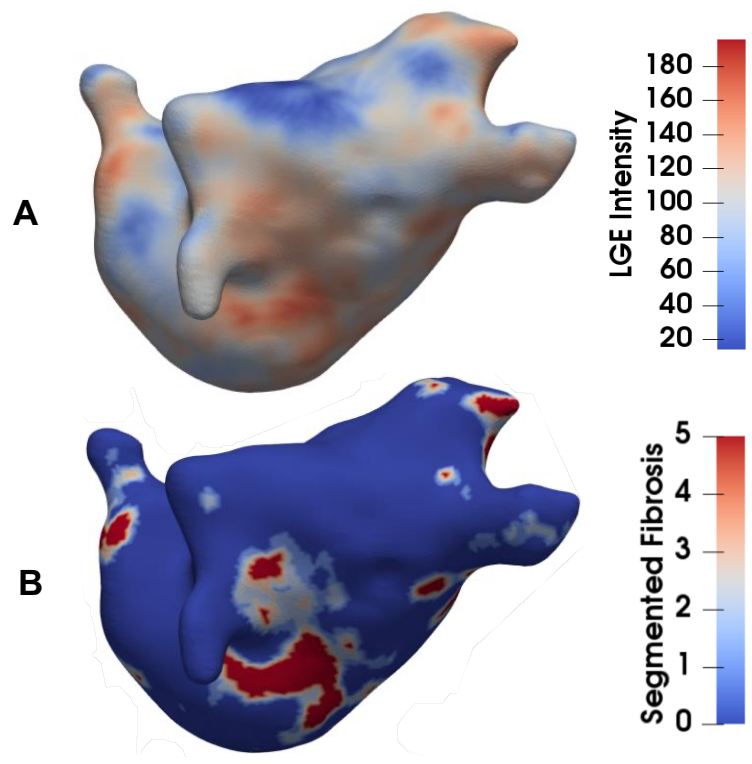

Figure 1. A: visualisation of the raw LGE signal intensity distribution in LA. B: the same LA after fibrosis is segmented (red) from healthy tissue (blue) using the IIR.

\subsection{Feature-Tracking}

Feature tracking algorithms can be used to extract the motion of structures through time for strain calculation.
The cyclic Cine MRI data had suitable temporal resolution of 50 time-steps to capture the cardiac cycle. Cine MRI data was processed using in-house software that generated deformation fields using an algorithm based on temporally-sparse free-form deformations [6]. The deformation field at each time step was then applied to the triangular LA surface meshes created from the manual segmentations and the coordinate location of each node was updated accordingly to generate a sequence of 50 time-dependant meshes which correspond to the motion of the LA during the cardiac cycle.
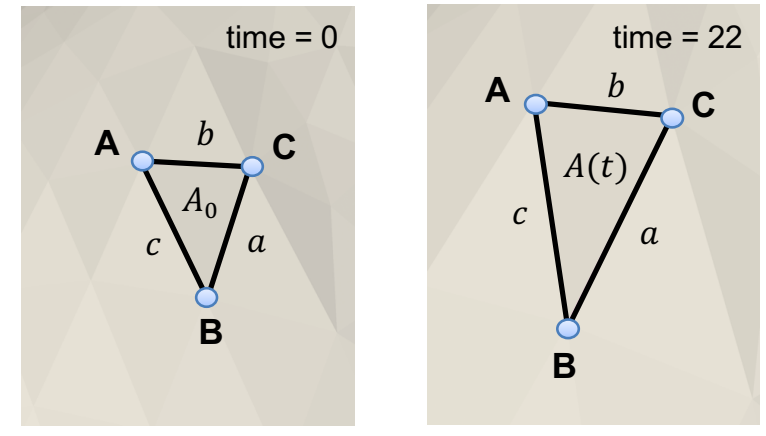

Figure 2. Magnified view of a surface triangle element at two different time-steps to visualise mesh deformation.

\subsection{Strain Calculation}

The deformed LA surface meshes were used to calculate the myocardial area strain (MAS). The coordinates of three nodes which form a triangle were used to obtain the length of sides $a, b$ and $c$ (Figure 2). These were used to find the semi-perimeter, $s$ (Eq. 1), and then in Heron's Formula (Eq. 2) to find the initial area of the triangle element $A_{0}$. This calculation was repeated for area of all elements on the LA surface mesh at every time-step, $A(t)$. Area strain, $\boldsymbol{\epsilon}(t)$, was calculated as the ratio of change in element area to its initial area (Eq. 3).

$$
\begin{gathered}
s=(a+b+c) / 2 \\
A=\sqrt{s(s-a)(s-b)(s-c)} \\
\boldsymbol{\epsilon}(t)=\frac{A(t)-A_{0}}{A_{0}}=\frac{\Delta A}{A_{0}}
\end{gathered}
$$

The maximum absolute MAS value for each element over the cardiac cycle was chosen to represent the largest achievable contractile motion of the LA under normal and damaged conditions. This metric was then mapped to the 3D LA meshes (Figure 3).

\subsection{D LA Unwrapping}

Regional analyses of MAS and fibrosis were enhanced by unwrapping the 3D LA models to a standardised 2D 
disk representation (2D LA) [7]. The technique averaged MAS and fibrosis values from the 3D model into 24 regions of clinical interest: 7 regions were defined on the LA body, 4 surrounding each of the four PVs and 1 for the left atrial appendage, while the mitral valve became the circumference of the 2D LA (Figure 4). The average value within each region was stored for further analysis.
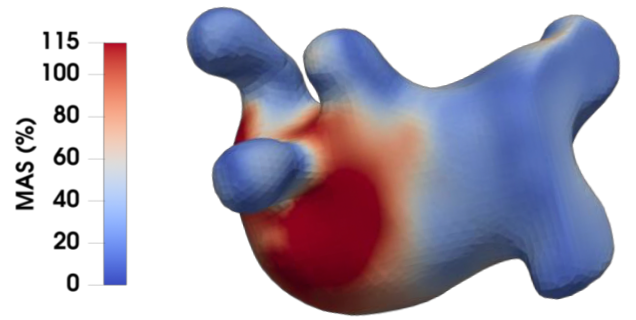

Figure 3. A 3D patient-specific LA mesh with maximum absolute MAS results mapped. Red regions indicate greater contractile motion than blue.

\section{Results}

\subsection{SR Group}

All patients in the SR group had increased fibrosis (mean $=0.4 \pm 0.3$ ) but showed an average decrease in MAS (mean $=-10 \% \pm 9.7$ ) over the LA after CA therapy. 2D disk results for one of the five SR patients are shown

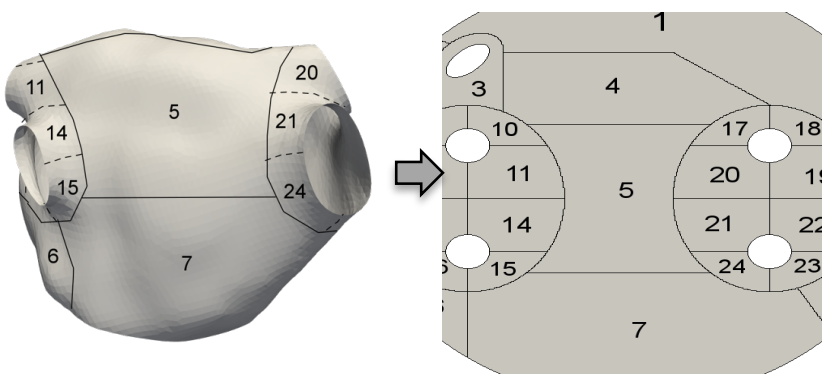

Figure 4. MRI-derived 3D LA surface with 24 regions labelled and corresponding locations on the 2D LA.

below in Figure 5. More red shades (fibrosis) are seen after $\mathrm{CA}$ in regions 15, 17, 18 and 24 which surround the PVs (Figure $5 \mathbf{A}, \mathbf{B}$ ). Conversely, regions 2, 6, 12 and 15 were bluer, indicating decreased MAS and contractility after CA (Figure $5 \mathbf{C}$ and D). A Spearman's rank correlation of $r_{s}=-0.52$ between fibrosis and MAS was found in region 5 - the LA posterior wall.

\subsection{AF Group}

Similar to the SR group, all patients in the AF group saw increases in fibrosis (mean $=0.4 \pm 0.3$ ), however MAS was improved in all regions (mean $=8.9 \% \pm 5.7$ ). A single patient is shown from the AF group in Figure 5. Changes in fibrosis (Figure $5 \mathbf{E}, \mathbf{F}$ ) show more redness in

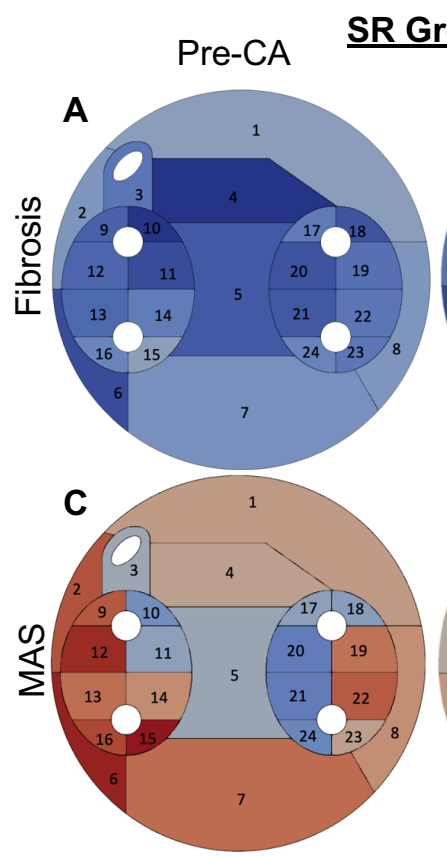

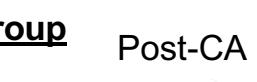
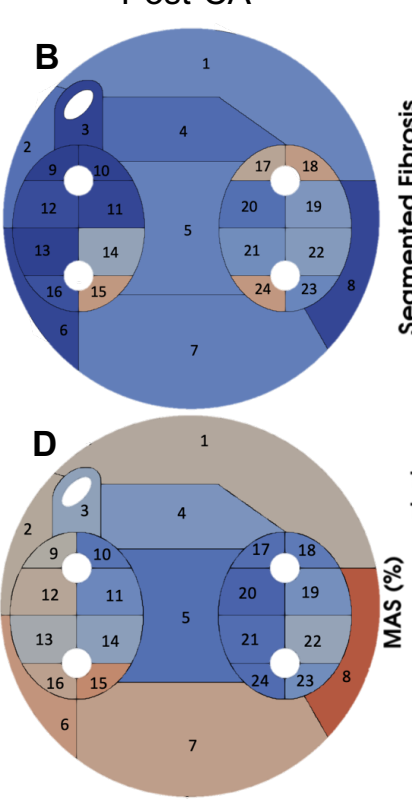

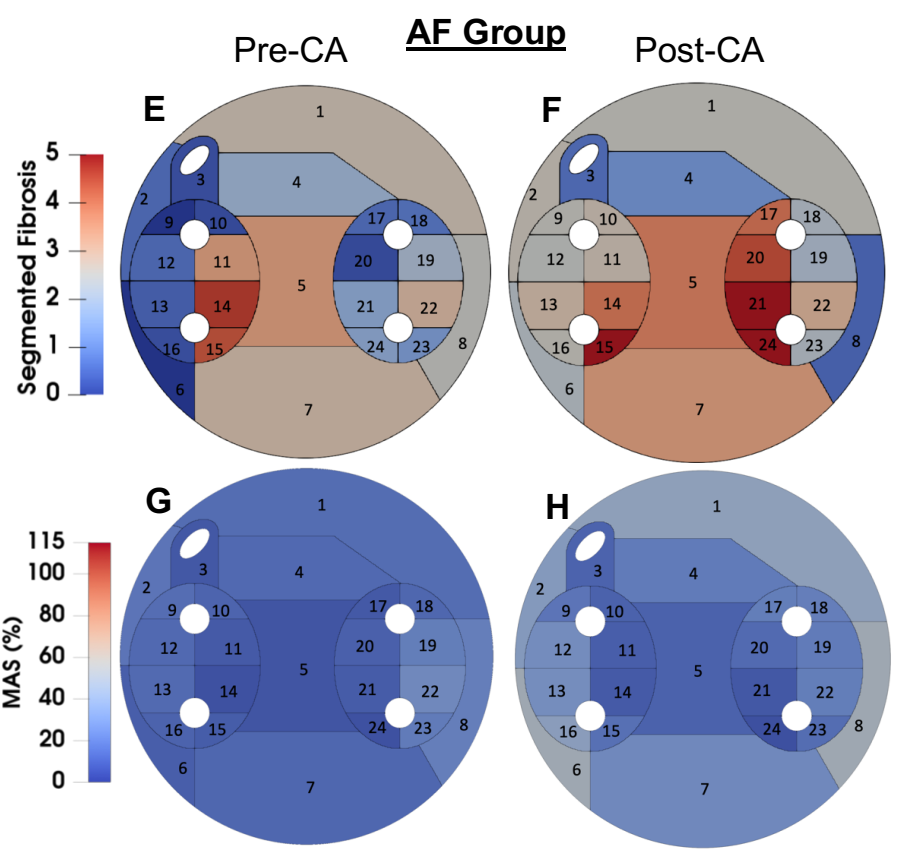

Figure 5. 2D LA disk representations of regional average fibrosis (top row) and MAS (bottom row) for a patient in the SR (A-D) and AF (E-H) groups. Pre-CA disks are on the left (A, C and $\mathbf{E}, \mathbf{G})$ and post-CA on the right (B, $\mathbf{D}$ and $\mathbf{F}, \mathbf{H})$. Colour scales for fibrosis and MAS are provided. 
regions 2, 6, 9, 12, 13, 19 and 16 near the left PVs and 17, 20 , and 24 at the right PVs after CA. The MAS disks (Figure $5 \mathbf{G}, \mathbf{H}$ ) present fewer blue shades in most regions of the 2D LA indicating greater LA motion after CA. Spearman's rank coefficients of $r_{s}=-0.99,-0.89$ and -0.71 were found in the LA posterior wall, left superior PV and LA floor, respectively, showing correlation between high level of fibrosis and low MAS.

\section{Discussion}

The results of this study show that myocardial strain can be used as a biomarker for atrial fibrosis as seen by identification of fibrotic scar created through PV isolation by $\mathrm{CA}$ in regions 9 to 23 in Figure 5. The SR group showed a decrease in MAS after CA. This is a novel and unexpected result as CA aims to restore LA function through terminating AF. However, CA of the myocardium to create scar tissue may reduce LA contractile ability.

Fibrosis distributions in Figure 5 show that the SR group had less fibrosis present in the LA than in AF group both pre- and post-CA, and had much higher MAS values throughout the LA. These results can mean that: 1) greater levels of fibrosis are linked to diminished contractile ability due to presence of more rigid collagen tissue, and 2) more fibrosis in the AF group could create more arrhythmogenic substrate resulting in increased likelihood of arrhythmic events, as predicted by modelling [5].

$\mathrm{AF}$ is widely recognised to significantly increase the risk of stroke [8]. The findings in this investigation can be coupled with simulations of LA fluid dynamics to greater understand the effect of reduced contractile motion on blood flow and coagulation to determine a personalised risk of stroke due to AF. Fibrosis and scar tissue will play an important part in this proposed research, as CA may cure AF by reverting to SR, but the remaining fibrosis may permanently detriment LA mechanical function.

This proof-of-concept research was performed with a small cohort size of $\mathrm{N}=8$, which may explain the high correlation observed in the AF group $(\mathrm{N}=3)$. These findings warrant further investigation on a larger cohort.

Additionally, other factors such as variable atrial wall thickness [9] and electrophysiological heterogeneity [10] can also contribute to regional differences in atrial mechanical function. Novel computational modelling and image processing techniques are being developed in our group to quantify the effect of these factors in the future.

In conclusion, this study created a novel pipeline for studying the effects of AF and fibrosis on LA mechanics assessed through MRI-derived strains. Our results confirm that 1) LA strain can be used as a biomarker for fibrosis and 2) CA therapy for AF can improve LA electrical function at the expense of having a detrimental effect on its mechanical function.

\section{Acknowledgments}

This research was supported by the EPSRC [EP/R513064/1].

\section{References}

[1] S. Nattel, "New Ideas about Atrial fibrillation 50 Years on," Nature, vol. 415, no. 6868, pp. 219-226, Jan. 2002.

[2] A. Roy et al., "Virtual Catheter Ablation of Target Areas Identified from Image-Based Models of Atrial Fibrillation," in FIMH 2019, Lecture Notes in Computer Science, vol. 11504 LNCS, pp. 11-19, 2019.

[3] H. Chubb et al., "The Reproducibility of Late Gadolinium Enhancement Cardiovascular Magnetic Resonance Imaging of Post-Ablation Atrial Scar: A Cross-Over Study," J. Cardiovasc. Magn. Reson., vol. 20, no. 1, p. 21, Dec. 2018.

[4] D. Dillon-Murphy et al., "Modeling Left Atrial Flow, Energy, Blood Heating Distribution in Response to Catheter Ablation Therapy," Front. Physiol., vol. 9, p. 1757, Dec. 2018.

[5] A. Roy, M. Varela, and O. Aslanidi, "Image-Based Computational Evaluation of the Effects of Atrial Wall Thickness and Fibrosis on Re-entrant Drivers for Atrial Fibrillation.," Front. Physiol., vol. 9, p. 1352, 2018.

[6] E. Kerfoot et al., "Eidolon: Visualization and Computational Framework for Multi-Modal Biomedical Data Analysis." LNCS, Vol. 9805 (Springer), 425-437

[7] S. E. Williams et al., "Standardized Unfold Mapping: a Technique to Permit Left Atrial Regional Data Display and Analysis.," J. Interv. Card. Electrophysiol., vol. 50, no. 1, pp. 125-131, Oct. 2017.

[8] P. A. Wolf, R. D. Abbott, and W. B. Kannel, “Atrial Fibrillation as an Independent Risk Factor for Stroke: The framingham study," Stroke, vol. 22, no. 8, pp. 983988, 1991.

[9] M. Varela et al., "Novel MRI Technique Enables NonInvasive Measurement of Atrial Wall Thickness," IEEE Trans. Med. Imaging, vol. 36, no. 8, pp. 1607-1614, Aug. 2017.

[10] M. Varela, M. A. Colman, J. C. Hancox, and O. V. Aslanidi, "Atrial Heterogeneity Generates Re-entrant Substrate during Atrial Fibrillation and Anti-arrhythmic Drug Action: Mechanistic Insights from Canine Atrial Models," PLOS Comput. Biol., vol. 12, no. 12, p. e1005245, Dec. 2016.

\section{Address for correspondence:}

Ahmed Qureshi; School of Biomedical Engineering and Imaging Sciences, King's College London, $3^{\text {rd }}$ Floor Lambeth Wing, St Thomas' Hospital, London, SE1 7EH, UK; E-mail: ahmed.qureshi@kcl.ac.uk 